\title{
Molecular analysis of the swallow gene of Drosophila melanogaster
}

\author{
Edwin C. Stephenson, Yu-Chan Chao, ${ }^{1}$ and James D. Fackenthal ${ }^{2}$ \\ Department of Biology, University of Rochester, Rochester, New York 14627 USA
}

\begin{abstract}
We report the cloning and molecular characterization of the swallow gene, a maternal-effect gene of Drosophila melanogaster. Maternal-effect genes are transcribed only during oogenesis; one such gene, bicoid, encodes a message that is localized at the anterior end of oocytes, eggs, and early embryos. swallow ${ }^{+}$activity is necessary during oogenesis for bicoid message localization. We show here that a fragment of a previously identified clone, introduced into the genome by P-element-mediated transformation, is able to rescue the maternal-effect lethality of swallow mutants, confirming the identity of this clone as swallow. By in situ hybridization, we show that swallow message is synthesized in nurse cells during oogenesis but is not localized along either the anterior-posterior or dorsal-ventral axes. We have confirmed an earlier finding that the localization of bicoid message is defective in swallow ${ }^{-}$oocytes and eggs.
\end{abstract}

[Key Words: Maternal-effect genes; RNA localization; oogenesis; embryonic polarity; in situ hybridization]

Received August 17, 1988; revised version accepted September 28, 1988.

In Drosophila, the specification of the embryonic axes utilizes information packaged into the egg during oogenesis (Anderson 1987; Nüsslein-Volhard et al. 1987). Anterior development relies on the product of the maternal-effect gene bicoid. bicoid- embryos (i.e., embryos whose mothers were homozygous for bicoid mutant alleles) lack most segmented head and thoracic structures, and the unsegmented portion of the head, the acron, develops as its posterior equivalent, the telson (Frohnhöfer and Nüsslein-Volhard 1986; Nüsslein-Volhard et al. 1987). bicoid ${ }^{-}$embryos can be rescued by injecting cytoplasm from a wild-type embryo. However, only cytoplasm from the extreme anterior tip of the embryo is effective, suggesting that the bicoid gene product is highly localized (Frohnhöfer and Nüsslein-Volhard 1986). The localization of bicoid activity results from the localization of bicoid mRNA at the anterior end of oocytes and embryos (Frigerio et al. 1986; Berleth et al. 1988). Two other maternal-effect genes, swallow and exuperatia, are necessary for the localization of bicoid activity; in $s w w^{-}$or $e x u^{-}$embryos, bicoid activity is not localized at the anterior end of the egg, as assayed in cytoplasmic transfer experiments (Frohnhöfer and Nüsslein-Volhard 1987), and bicoid message is distributed more or less uniformly (Berleth et al. 1988; this paper).

In this paper we report the cloning and initial characterization of swallow, one of the genes necessary for bi-

${ }^{1}$ Current address: Boyce Thompson Institute for Plant Research, Cornell University, Ithaca, New York 14853 USA.

${ }^{2}$ Current address: Department of Biology, Indiana University, Bloomington, Indiana 47405 USA. coid message localization. We previously described a molecular screen for clones of maternal-effect genes (Stephenson and Mahowald 1987) and presented evidence based on genomic proximity that one of these clones was the swallow gene. Here, we provide direct evidence that the clone identified previously contains the swallow gene and investigate the temporal and spatial expression of swallow RNA in oogenesis and embryogenesis. Also, we have examined the requirement for swallow ${ }^{+}$activity in bicoid message localization.

\section{Results \\ Mapping the position of the maternally restricted transcript}

In an earlier publication (Stephenson and Mahowald 1987) we described the isolation of genomic clone B70, which encodes part of a 2.1-kb RNA that appears to be restricted to the maternal RNA population. We also showed that both clone B70 and the maternal-effect locus swallow are located within the small deficiency $D f(1) J F 5$, suggesting that they represent the same gene. As a next step in the characterization of the maternal RNA encoded by clone B70, we isolated genomic clones adjacent to B70. The restriction map of a part of this walk is shown in Figure 1. Using probes from various parts of the walk for in situ hybridizations to the polytene chromosomes of larvae heterozygous for Df(1)JF5, we determined that the $28 \mathrm{~kb}$ of DNA represented in Figure 1 lies entirely within deficiency $D f(1)$ JF5 (data not shown).

To determine the position of the maternally restricted transcript, fragments of these clones were employed as 


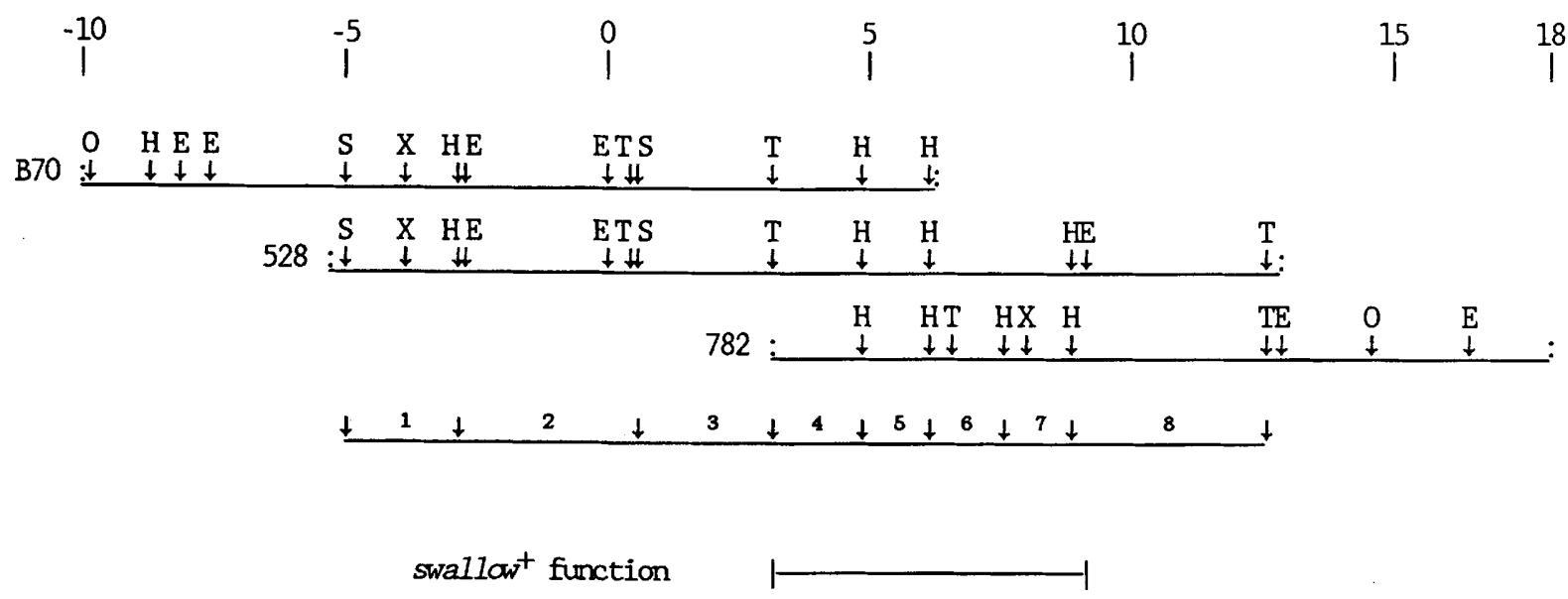

Figure 1. Molecular map of the swallow region. Restriction map of a 28 -kb region from polytene interval $5 \mathrm{E}$. The top line indicates the scale in kilobase pairs. Coordinate zero is the EcoRI site in B70. Restriction maps of genomic clones B70, 528, and 782 are shown on the next three lines. (E) EcoRI; (H) HindIII; (S) SaII; (T) SstI; (X) XbaI; (O) XhoI. An SstI site (position 6.6), a HindIII site (7.6), an XbaI site (8.0), and an EcoRI site (9.1) are polymorphic between the two strains from which these clones were derived, Canton $S$ for $\lambda B 70$ and $\lambda 782$, and a strain isogenized for a $c n b w$ second chromosome for $\lambda 528$. We have confirmed by Southern blot analyses that the polymorphisms present in these clones represent the positions of the sites in these strains accurately (data not shown). The fifth line indicates restriction fragments used as probes in the Northern blots shown in Fig. 2. The sixth line indicates the fragment that was found to provide complete swallow ${ }^{+}$function when introduced into the genome by P-element-mediated transformation.

probes in the Northern blot analyses shown in Figure 2a. The RNA of interest here, the 2.1-kb RNA that appears to be restricted to the maternal RNA population, is detected after hybridization with each of three HindIII fragments of sizes $1.3,1.5$, and $1.2 \mathrm{~kb}$, respectively, falling between map coordinates 4.8 and 8.8 (probes 5,6 and 7). The $2.1-\mathrm{kb}$ maternally restricted transcript is the most abundant RNA encoded by clone B70 /Stephenson and Mahowald 1987); its presence and relative abundance was the basis for our earlier identification of B70 as encoding a predominantly maternal RNA, despite the fact that B70 encodes other RNAs that are expressed at other developmental stages.

We determined the direction of transcription of the 2.1-kb maternally restricted RNA, using single-stranded RNA probes. The HindIII 1.3-kb fragment (probe 5 in Fig. 1) was cloned into the plasmid vector pGEM2 in both orientations. Each plasmid was linearized by digestion with EcoRI, which cuts only within the multiple cloning site of the plasmid vector, and ${ }^{32} \mathrm{P}$-labeled RNAs were synthesized using T7 RNA polymerase. As shown in Figure $2 b$, only one of these two probes hybridizes to the $2.1-\mathrm{kb}$ RNA. The orientation of the insert in this clone allows us to conclude that the direction of transcription of the 2.1-kb RNA is left to right, as the map is drawn in Figure 1.

RNAs expressed at most or all developmental stages are detected by other probes from the clones shown in Figure 1. A 2.1-kb RNA expressed at all embryonic stages is detected by a $2.2-\mathrm{kb}$ restriction fragment between coordinates -5.0 and -2.8 (probe 1). Despite the similarity in size, the $2.1-\mathrm{kb}$ RNA detected by probe 1 is not related to the $2.1-\mathrm{kb}$ maternally restricted RNA described above. A $1.8-\mathrm{kb}$ RNA is detected by probes be- tween coordinates -5.0 and -0.6 (probes 1 and 2). A pair of RNAs of sizes 3.8 and $3.4 \mathrm{~kb}$ are detected by probes between coordinates -5.0 and 4.8 (probes $1-4$ ). We have not investigated the organization of these RNAs; however, we assume that some of them are related, overlapping RNAs that differ in their initiation, termination, and/or splice sites. Such a relationship seems especially likely for the 3.8- and 3.4-kb RNAs, because each is detected by four adjacent probes. The 1.8 kb RNA may also be a variant of the $3.8 / 3.4-\mathrm{kb}$ RNAs because the three RNAs overlap in the -5.0 to -0.6 interval. To the right of the maternally restricted RNA a restriction fragment from clone 782 (probe 8 ) detects a 2.5-kb RNA. We showed previously that the 3.8-, 3.4-, and 1.8-kb RNAs are present in larval and pupal stages, in adult males and in nonovarian tissues of adult females (Stephenson and Mahowald 1987); we have not investigated the postembryonic expression of the $2.1-\mathrm{kb}$ RNA detected by probe 1 or the $2.5-\mathrm{kb}$ RNA detected by probe 8 .

\section{P-element-mediated transformation rescues swallow mutants}

The presence of the 3.8/3.4- and 2.5-kb transcripts was useful in defining the limits of the maternal gene: We assumed that the gene encoding the $2.1-\mathrm{kb}$ RNA and its regulatory sequences lay between the sequences encoding the 3.8/3.4-kb RNA upstream and the $2.5-\mathrm{kb}$ RNA downstream. Accordingly, we cloned the $5.9-\mathrm{kb}$ SstI-EcoRI fragment (coordinates 3.2-9.1) from $\lambda 528$ into the P-element transformation vector Carnegie 20 (Rubin and Spradling 1983), as described in Methods. This $5.9-\mathrm{kb}$ fragment contains the $4.0-\mathrm{kb}$ region shown 

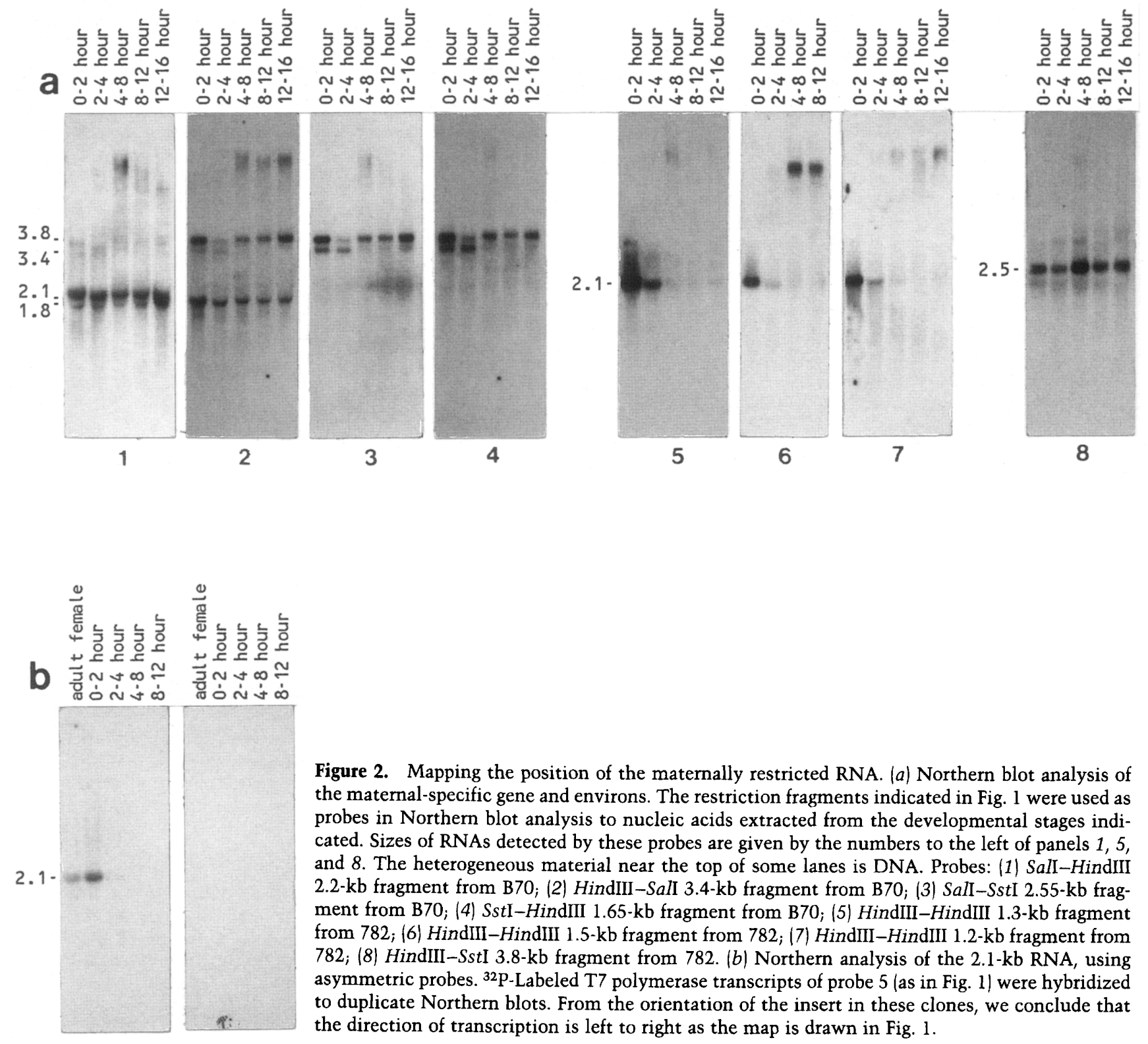

Figure 2. Mapping the position of the maternally restricted RNA. (a) Northern blot analysis of the maternal-specific gene and environs. The restriction fragments indicated in Fig. 1 were used as probes in Northern blot analysis to nucleic acids extracted from the developmental stages indicated. Sizes of RNAs detected by these probes are given by the numbers to the left of panels 1,5 , and 8 . The heterogeneous material near the top of some lanes is DNA. Probes: $(1)$ SalI-HindIII 2.2-kb fragment from B70; (2) HindIII-SalI 3.4-kb fragment from B70; (3) SalI-SstI 2.55-kb fragment from B70; (4) SstI-HindIII 1.65-kb fragment from B70; (5) HindIII-HindIII 1.3-kb fragment from 782 ; 6 ) HindIII-HindIII 1.5 -kb fragment from 782 ; (7) HindIII-HindIII 1.2-kb fragment from $782 ;(8)$ HindIII-SstI 3.8-kb fragment from 782 . (b) Northern analysis of the $2.1-\mathrm{kb}$ RNA, using asymmetric probes. ${ }^{32} \mathrm{P}$-Labeled $\mathrm{T} 7$ polymerase transcripts of probe 5 (as in Fig. 1) were hybridized to duplicate Northern blots. From the orientation of the insert in these clones, we conclude that the direction of transcription is left to right as the map is drawn in Fig. 1.

above to encode the maternally restricted $2.1-\mathrm{kb}$ RNA and $\sim 0.3 \mathrm{~kb}$ of downstream DNA. It also contains $1.6 \mathrm{~kb}$ of sequence upstream from the leftmost HindIII site and includes a portion of the DNA that encodes the 3.8/3.4kb RNA.

A mixture of this construct and the transposase helper plasmid p $\Pi 25.7 \mathrm{wc}$ (Karess and Rubin 1984) was injected into $r y^{-}$embryos, and $r y^{+}$flies in the G2 generation selected to begin lines. Lines in which $r y^{+}$segregated as an autosomal character were crossed into a $s w w^{-} r y^{-}$ background, and stocks of the genotype $s w w^{1497 /}$ $s w w^{1497} ; \mathrm{ry}^{506} / \mathrm{ry}^{506} ; \mathrm{P}\left[\left(\mathrm{ry}^{+}, s w w^{+}\right) t 528.11\right]$ were constructed and maintained. $r y^{+}$inserts on the $\mathrm{X}$ chromosome were crossed onto a $s w w^{11-999} \mathrm{X}$ chromosome and maintained in a $r y^{506} / \mathrm{ry}^{506}$ third chromosome background. The chromosomal positions of the P-element inserts were determined by in situ hybridization to polytene chromosomes and are shown in Table 1.

Restoration of swallow ${ }^{+}$function is scored easily as the survival of progeny past the first larval instar: Almost all progeny from swallow $w^{-}$females die as unhatched embryos, and the rest die as small first instar larvae (Stephenson and Mahowald 1987). For each of the seven lines containing a P-element-mediated insertion, the introduced $5.9-\mathrm{kb}$ fragment rescued the maternal-effect lethality of swallow. For five lines in which the P-element transposon was inserted on an autosome, we compared the development of embryos whose mothers were sisters of the genotypes $s w w / s w w ; r y / r y$ and $s w w /$ sww; ry/ry; $\mathrm{P}\left[\left(\mathrm{ry}^{+}, s w w^{+}\right)\right.$t528.11]. All progeny produced by $\mathrm{ry}^{-}$females died with the lethal swallow phenotype. However, $r y^{+}$females produced viable embryos 
Table 1. P-element transformation rescues swallow

\begin{tabular}{ccc}
\hline Stock & $\begin{array}{l}\text { Chromosomal } \\
\text { position }\end{array}$ & $\begin{array}{l}\text { Survival } \\
(\%)\end{array}$ \\
\hline bb154 & $73 \mathrm{BC}$ & 98.1 \\
bb275 & $46 \mathrm{CD}$ & 97.0 \\
bb344 & 53BC & 81.0 \\
bb421 & $7 \mathrm{C}$ & 90.1 \\
bb524 & 1F & 94.1 \\
bb635 & $45 \mathrm{CD}$ & 93.5 \\
bb854 & $94 \mathrm{EF}$ & 97.4 \\
\hline
\end{tabular}

Stock numbers for seven lines of flies transformed with P-element construct $\mathrm{t} 528.11$ are given in the first column. The chromosomal site of insertion, as determined by in situ hybridization to polytene chromosomes, is given in the second column. The third column presents measurements of the survival to the second larval instar of progeny from females of the genotype

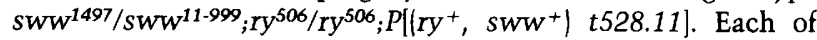
seven different sites of insertion rescues the maternal-effect lethality of flies whose resident swallow genes are both mutant.

at a frequency equivalent to that of wild-type flies (Table 1). For the two lines in which the $r y^{+}$construct was inserted on the $\mathrm{X}$ chromosome, we recombined the P-element insert onto a $s w w^{-} \mathrm{X}$ chromosome and found that the P-element insert was able to rescue the maternal-effect lethality of swallow. For each of these X chromosome lines, we subsequently removed the $r y^{+} s w w^{+} \mathrm{P}$ element by recombination to confirm that the resident copy of swallow was a mutant allele.

For six of the seven lines, $>90 \%$ of the embryos produced by transformed females survived to the second larval instar (Table 1). This value is similar to the survival of the progeny of wild-type flies tested under the same conditions. Surviving larvae were morphologically normal, and virtually all developed into normal adults. Of the progeny that failed to reach the second larval instar, none developed with a swallow ${ }^{-}$phenotype, and most appear to have been unfertilized eggs. For the seventh line, $b b 344$, only $\sim 80 \%$ of the progeny developed normally. However, the chromosome containing this insert is homozygous lethal and confers a weak dominant Minute phenotype; both phenotypes are apparently associated with the swallow ${ }^{+}$P-element-mediated insert. We believe that the lower survival rate of these embryos resulted from a generally lower viability in this stock and is not related to swallow function. As was true for the other lines, none of the offspring that died before the second larval instar had a swallow ${ }^{-}$phenotype.
Synthesis and accumulation of swallow RNA during oogenesis and embryogenesis

We determined the spatial pattern of expression of swallow message in oogenesis and embryogenesis by in situ hybridization to sectioned ovaries and embryos (Fig. 3; see Methods|. Oogenesis begins with four incomplete divisions of a cytoblast that generates a cyst of 16 interconnected cells, one of which becomes the oocyte, and the other 15 of which become nurse cells. The 15 nurse cells, the oocyte, and a surrounding layer of follicle cells of somatic origin are known as the egg chamber. We are able to detect swallow message reliably in stage-3 egg chambers; younger stages have lower autoradiographic signals that are only slightly above background levels [Fig. 3a; for a description of oogenesis and its morphological stages, see Mahowald and Kambysellis (1980)]. The amount of swallow message increases in previtellogenic stages, reaching its highest concentration in the nurse cell complex of stage-10 egg chambers. In early stages, swallow message appears to be distributed uniformly in the oocyte-nurse cell complex and is absent from the surrounding follicle cells. As the egg chamber grows during these previtellogenic stages, the concentration of swallow message increases substantially, but its level remains approximately equivalent in nurse cells and in the oocyte proper. In vitellogenic stages of Drosophila oogenesis, the oocyte grows through the accumulation of yolk, synthesized in fat bodies and in the follicle cells. By stage 10 (Fig. 3b) when the oocyte occupies about half of the egg chamber, the concentration of swallow message is clearly higher in nurse cells than in the oocyte. Within nurse cells, the concentration of swallow message is uniform except for the nurse cell nuclei, which have substantially lower levels. In stages of oogenesis following that shown in Figure $3 \mathrm{~b}$, the nurse cells empty their cytoplasmic contents into the oocyte. The end product of this process, the stage-14 oocyte shown in Figure $3 \mathrm{c}$, has a uniform distribution of swallow message.

Early embryogenesis involves the rapid cleavage of zygotic nuclei and their migration to the periphery of the embryo. In cleavage-stage embryos, swallow message is distributed uniformly along anterior-posterior and dorsal - ventral axes (Fig. 3d). As the nuclei migrate to the periphery of the embryo, swallow message becomes most highly concentrated in the nonyolky peripheral cytoplasm that surrounds the nuclear layer (Fig. 3e). The amount of swallow message in the embryo is reduced during the transition from the cleavage stage to the blas-

Figure 3. swallow message accumulation in ovaries and embryos. swallow mRNAs were detected by in situ hybridization to sections of wild-type ovaries and embryos. Bright-field (left) and dark-field (right) micrographs are shown. The bar in $a$ represents $100 \mu \mathrm{m}$. Exposures for $a$ and $b$ were 8 days and for $c-f 6$ days. (a) A cluster of previtellogenic egg chambers. Stages of oogenesis, as described in Mahowald and Kambysellis (1980), are indicated by the numbers. swallow message concentration increases in these early stages, reaching its highest level in the nurse cells of stage-10 egg chambers (lower left and lower right; see also $b)$. $(b)$ Stage-10 egg chamber. Stage-10 nurse cells contain the highest concentration of swallow message, which is several times higher than in stage-10 oocytes. swallow RNA is absent from follicle cells at this and all other stages of oogenesis. $(\mathrm{N})$ Nurse cell complex; $(\mathrm{O})$ oocyte; $(\mathrm{F})$ follicle cells. (c) Stage-14 oocyte. swallow message is uniform in its distribution. (d) Preblastoderm embryo. swallow message is uniformly distributed. (e) Syncytial blastoderm embryo. swallow message is more highly concentrated at the embryonic periphery. $(f)$ Cellular blastoderm embryo. The small amount of swallow message still detectable is in the cellularized periphery of the embryo. 
toderm stage (cf. Fig. 3d and e). The micrographs shown in Figure 3, $d-f$, are from the same experiment and are the same autoradiographic exposure, so the decrease in signal observed at successive embryonic stages roughly represents the actual decrease in swallow message levels. During cellularization of the blastoderm (Fig. 3f), the concentration of swallow message is reduced further, although that remaining is concentrated in the pe-

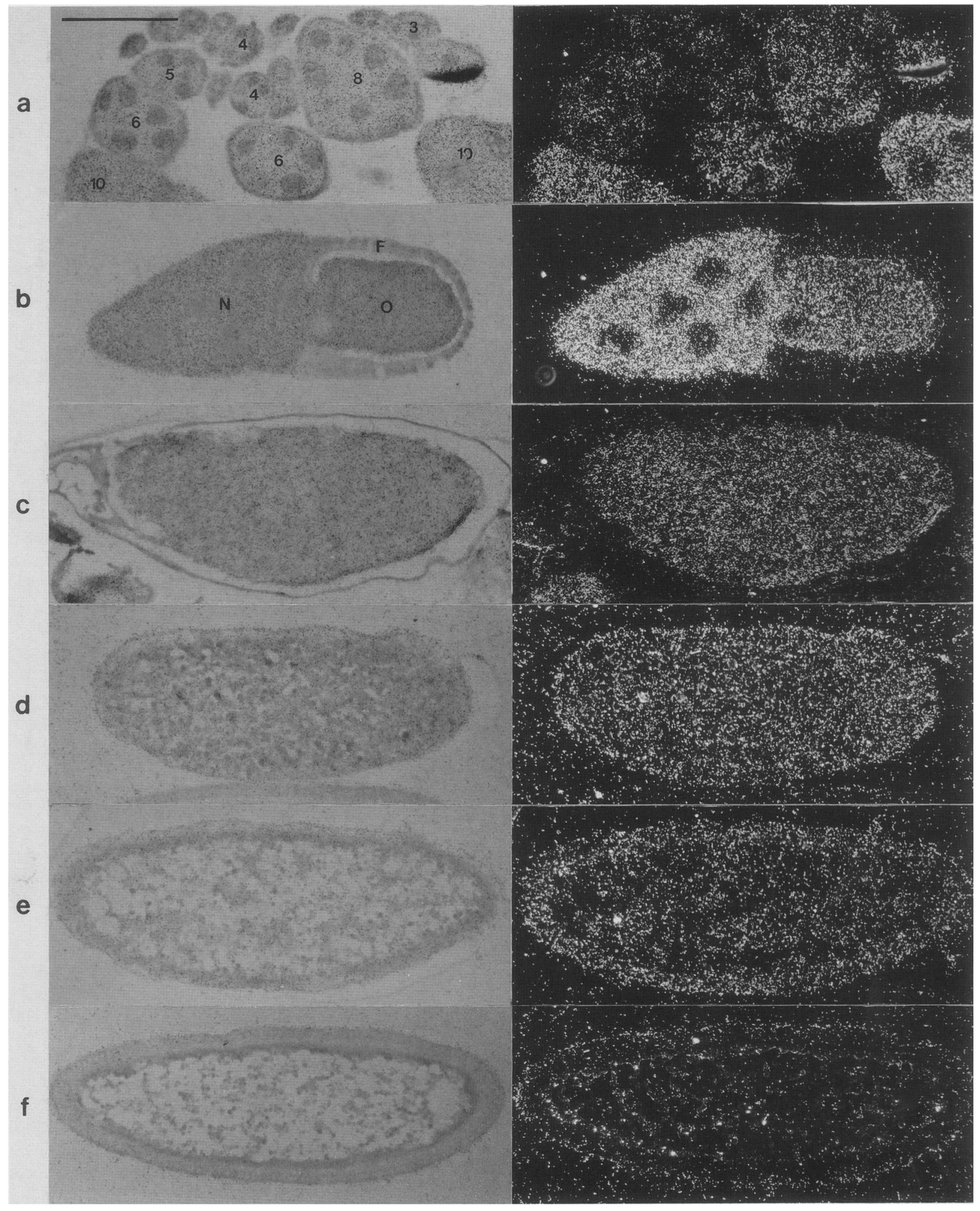

Figure 3. (See facing page for legend.) 
ripheral cytoplasm. swallow message can no longer be detected by the beginning of gastrulation (not shown).

The uniform distribution of swallow message levels in oocytes and early embryos raises the concern that these hybridization results might be due to a nonspecific association of the probe with the tissue. As a control for the specificity of swallow message hybridization, we hybridized sense strand RNA probes to early embryos; there is no detectable signal using such a probe (not shown). In addition, although swallow message levels are uniform in oocytes and early embryos, the absence of a hybridization signal in other tissues (follicle cells, nurse cell nuclei, postblastoderm embryos) argues against such a nonspecific association.

bicoid message localization in wild-type and swallow ${ }^{-}$ egg chambers

As reported previously (Frigerio et al. 1986; Berleth et al. 1988), bicoid message is localized to the anterior end of oocytes and early embryos. Examples of the localization pattern in wild-type egg chambers are shown in Figure 4. Figure 4a shows the distribution of bicoid message in a stage-10 wild-type egg chamber, where it is present in the nurse cell complex and localized to the anterior margin of the oocyte, the part of the oocyte closest to the nurse cells. bicoid message is clearly nonuniform, or 'patchy,' in its distribution in nurse cell cytoplasm, in contrast to the relatively uniform distribution of swallow message in nurse cell cytoplasm /cf. Figs. 3b and $4 \mathrm{a}$ ). bicoid message remains restricted to the anterior oocyte margin in subsequent stages, as in the fullgrown stage-14 oocyte shown in Figure $4 \mathrm{~b}$.

bicoid message distribution in swallow ${ }^{-}$oocytes is abnormal, with defects that become apparent during vitellogenic stages of oogenesis. In stage-10 egg chambers (Fig. 4c) bicoid message is restricted to the oocyte margin, as in wild-type oocytes at this stage. However, in contrast to the strict anterior localization observed in wild-type oocytes, bicoid message is most highly concentrated in lateral regions of swallow ${ }^{-}$oocytes (arrows in Fig. 4c). Older oocytes show a progressive degeneration of the localization pattern, as in the stage-12 swallow $w^{-}$oocyte shown in Figure $4 \mathrm{~d}$ : bicoid message is still highly concentrated in the oocyte periphery, but this peripheral localization includes the anterior twothirds of the oocyte surface. In addition, there are significant levels of bicoid message in internal parts of the oocyte. Finally, in mature swallow ${ }^{-}$oocytes (Fig. 4e), bicoid message is only slightly more concentrated at the oocyte periphery than in internal regions and is distributed in a shallow anterior-posterior gradient, with the anterior end of the oocyte having the highest bicoid message levels. The micrographs shown in Figure 4, $\mathrm{c}-\mathrm{e}$, are from a different experiment and a shorter autoradiographic exposure than those shown in Figure 4, a and $b$, so differences in signal intensity in Figure 4 do not indicate differences in bicoid message levels in wild-type and swallow egg chambers. On the contrary, we believe that bicoid message levels in swallow egg chambers are approximately the same as in wild-type egg chambers, based on visual comparison of in situ hybridization signals from an experiment where wild-type and swallow slides had the same exposure time /data not shown). We have also examined bicoid message levels by dot blot analysis and found that message levels are approximately the same in wild-type and swallow ovaries (N.J. Pokrywka and E.C. Stephenson, unpubl.).

\section{Discussion}

We report here the initial molecular characterization of the maternal-effect gene swallow of Drosophila melanogaster. We show that a cloned fragment is able to rescue the maternal-effect lethality of swallow alleles, proving that a $2.1-\mathrm{kb}$ maternally restricted transcript encoded therein is the transcript of the swallow gene. swallow was identified originally in genetic screens for female sterile and maternal-effect mutations /Gans et al. 1975; Mohler and Carroll 1984; Stephenson and Mahowald 1987). All known alleles of swallow are homozygous and hemizygous viable, and males have normal fertility. However, females produce only defective embryos, most of which die at the end of embryogenesis with defects in the cephalopharyngeal apparatus, the anterior-most part of the embryonic body plan. From its genetic behavior, swallow has been classified as a maternal-effect lethal, a gene whose expression should be limited to oogenesis. We showed previously by Northern blot analysis that swallow message is detectable only in the ovaries of adult females and in very early embryos (Stephenson and Mahowald 1987). In situ hybridization experiments presented here extend these observations, showing that nurse cells and the oocyte contain high levels of swallow message but that swallow message is undetectable in follicle cells. These results are consistent with genetic experiments showing that swallow ${ }^{+}$activity is necessary in the germ-line portion of the ovary (i.e., nurse cells and the oocyte) but not necessary in ovarian tissues of somatic origin (i.e., follicle cells) (Perrimon and Gans 1983). Furthermore, in situ hybridization experiments shown here indicate that high levels of swallow message are present in the newly fertilized egg but decrease rapidly during the blastoderm stages of early embryogenesis. Because swallow message levels begin to decrease at the time when the zygotic genome first becomes active (the syncytial blastoderm stage; $\mathrm{Za}$ lokar 1976; Edgar and Schubiger 1986), we think it unlikely that transcription from the zygotic genome contributes significantly to swallow message levels in the early embryo, once again as would be expected for a strict maternal-effect gene.

Data presented here show that a $5.9-\mathrm{kb}$ cloned fragment rescues the maternal effect lethality of swallow. Seven independent lines, each with a different genomic site of insertion for swallow ${ }^{+}$, were effective; in this small sample we did not find a genomic position that allowed rosy ${ }^{+}$expression but failed to permit expression of swallow ${ }^{+}$sufficient to rescue the maternal-effect lethality of swallow mutants. Although these data indi- 


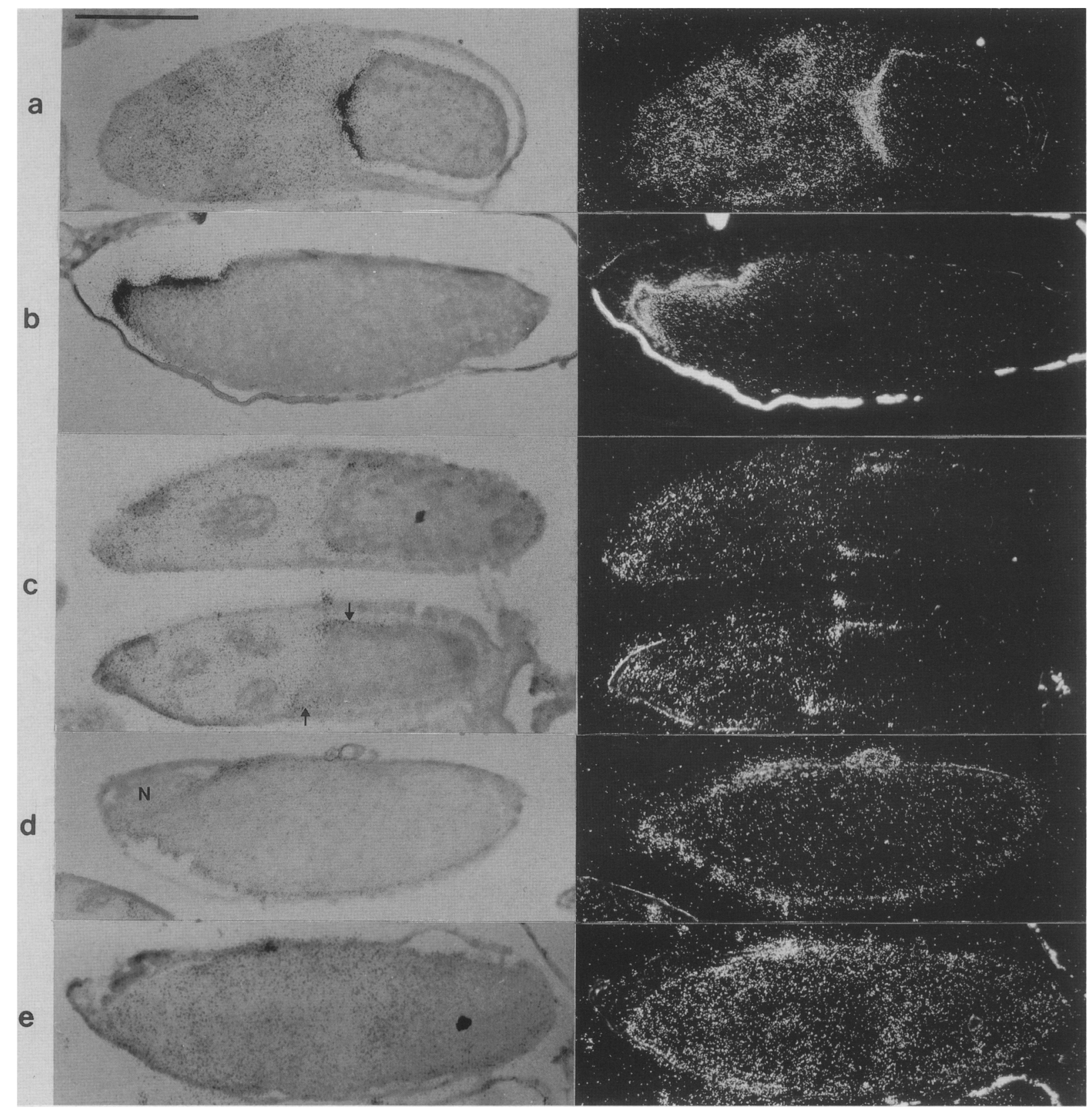

Figure 4. bicoid mRNAs in wild-type and swallow ${ }^{-}$egg chambers. ${ }^{35}$ S-Labeled bicoid RNA probes were hybridized to sectioned ovaries as described in Methods. Bright-field (left) and dark-field (right) micrographs are shown. The bar in $a$ represents $100 \mu \mathrm{m}$. Exposure time for $a$ was 13 days and for $b-e 6$ days. (a) Stage-10 wild-type egg chamber. bicoid message accumulates in the oocyte at its nurse cell-proximal margin. (b) Stage-14 wild-type oocyte. bicoid message is prominent at the anterior margin of the mature oocyte. (c) Stage-10 swallow $w^{-}$egg chambers. bicoid message is predominantly localized to the oocyte margin, but to the lateral rather than the anterior margin of the oocyte (arrows). (d) Stage-12 swallow ${ }^{-}$oocyte. Much of the bicoid message is localized at the periphery of the oocyte, although some is internal as well. Degenerating nurse cells $(\mathrm{N})$ contain no bicoid message. (e) Stage-14 swallow $w^{-}$oocyte. The peripheral concentration of bicoid message seen in earlier stages is reduced, and bicoid message is distributed more or less uniformly on the anterior-posterior axis, with a slightly higher concentration at the anterior end of the egg.

cate the molecular identity of the swallow gene, they do not address the question of whether the P-element-inserted copies of swallow are expressed correctly. We have not attempted to quantitate the level or timing of expression from the P-element-inserted copies, nor do we know what level of swallow ${ }^{+}$expression is required to rescue the maternal-effect lethality of the swallow mutants we employed in these studies.

Despite its role in the bicoid message localization process, swallow message is not itself localized along the anterior-posterior axis. The distribution of swallow message is notable, however, in a few respects. First, in 
vitellogenic stages of oogenesis, there are higher concentrations of swallow message in the nurse cell complex than in the oocyte itself. This accumulation in nurse cells may reflect a requirement that most of the swallow protein be translated in nurse cells and not in the oocyte. As an alternative, swallow message accumulation may reflect the kinetics of synthesis and export: Nurse cell nuclei may be synthesizing swallow message at a rate that exceeds the rate of transport into the oocyte. Second, in blastoderm embryos swallow message distribution changes from a uniform pattern to one in which it is more highly concentrated at the embryonic periphery. Because this redistribution is accompanied by a reduction in the quantity of swallow message in the embryo, we do not know whether this change reflects the movement of swallow message, its selective degradation in the center of the embryo, or a combination of both mechanisms.

One of the roles of swallow is to localize or maintain the localization of bicoid message. Frohnhöfer and Nüsslein-Volhard (1987) inferred such a role for swallow and exuperantia because they were unable to find high concentrations of bicoid ${ }^{+}$rescuing activity at the anterior tip of $s w w^{-}$and $e x u^{-}$early embryos. This observation has since been confirmed at the molecular level by Berleth et al. (1988) and for swallow in the experiments reported in this paper. One can envision two general classes of models for the mechanism of bicoid message localization during oogenesis. In the first, cytoplasm at the anterior end of the egg is different from cytoplasm elsewhere, and bicoid message is localized by a homing or transport mechanism that depends on this preexisting molecular inhomogeneity. In the second model, oocyte cytoplasm is uniform, with the capacity to localize bicoid message everywhere: bicoid message is 'trapped' as it enters the oocyte from the nurse cells, and the anterior localization of bicoid message results simply from the anterior location of nurse cells. We favor the second model for the simple way in which the inherent polarity of the egg chamber is translated into embryonic polarity. In support of the second model, the studies reported here indicate that swallow message is not itself localized or concentrated along the anterior-posterior axis. Of course, the observations on swallow do not directly invalidate the first model described above: swallow protein may be localized even though its message is not; or another component of the bicoid message localization machinery may be localized, whereas the swallow RNA and protein are distributed uniformly.

We show here that the failure to localize bicoid message in swallow ${ }^{-}$oocytes is not absolute: In early vitellogenic stages, bicoid message is localized; but as oogenesis proceeds, the localization deteriorates. The significance of the transient bicoid message localization is not clear, but it is interesting to speculate that it reflects the role of the swallow gene product in this process. The swallow ${ }^{+}$protein may, for example, cross-link or stabilize the cytoskeletal elements to which bicoid message is presumably attached. In this case, one might imagine that the initial localization of bicoid message in swallow $w^{-}$oocytes results from the near-normal assembly of a cytoskeletal system and that the localization deteriorates later in the absence of a swallow ${ }^{+}$-dependent stabilization of this cytoskeletal network. As noted by others (Frohnhöfer and Nüsslein-Volhard 1987; Berleth et al. 1988), a role for swallow in cytoskeletal function is attractive because some of the embryos from swallow $w^{-}$mothers are abnormal with respect to nuclear migration and cellularization of the blastoderm, processes that probably require cytoskeletal functions. On the other hand, it is conceivable that the partial loss of bicoid message localization may result from partial loss of swallow ${ }^{+}$function in the mutants employed in the in situ hybridization experiments. The animals used here were heterozygous for a strong swallow allele $\left(s w w^{11-999}\right)$ and a deficiency for the locus, $D f(1) J F 5$, the strongest phenotypic combination available to us. We have observed the same gradual loss of bicoid message localization, using flies heterozygous for another strong allele, $s w w^{1497}$, and the deficiency (E.C. Stephenson, unpubl.). However, we are not certain that these genotypic combinations represent the amorphic condition and cannot rule out the possibility that the transient localization of bicoid message in these oocytes reflects residual $s w w^{+}$ function.

The eggs of many animals are nonhomogeneous, with distinctive cytoplasmic domains or organelles localized in particular regions. These inhomogeneities may exist prior to fertilization or may come about through a cytoplasmic rearrangement shortly after fertilization. In some cases, it has been shown that the segregation of a particular cytoplasmic domain into a subset of blastomeres during the early embryonic cleavages is necessary and sufficient for the development of particular embryonic tissues. The pole plasm in Drosophila, the polar granules in Caenorhabditis elegans, the yellow crescent in ascidians, and the polar lobe cytoplasm of mollusk and annelid eggs are well-characterized examples (for review, see Davidson 1986). Some of these systems have been studied for many years, yet there are only a few studies on the cellular mechanisms of cytoplasmic localization in eggs and cytoplasmic reorganization in embryos. In ascidian embryos, the yellow pigment granules appear to be associated with a cortical actin-rich cytoskeleton (Jeffrey and Meier 1983), and the concentration of these granules into the yellow crescent requires actin filament function (Zalokar 1974). In C. elegans, the P granules coalesce and segregate into the posterior P1 cell at the first cleavage; this movement requires actin function (Strome and Wood 1983). In Xenopus embryos, specification of the dorsoventral axis involves a signal that emanates from the vegetal pole, and its transport to or utilization by the equatorial mesoderm requires microtubule function (Scharf and Gerhart 1983). The conventional view has been that molecules or organelles necessary for particular developmental decisions are bound in the specialized cortical cytoplasm of the egg, a region devoid of yolk granules and lipid droplets and relatively rich in cytoskeletal elements (for review, see $\mathrm{Da}$ vidson 1986). However, this cortical cytoskeleton is not 
well characterized, and the mechanisms by which molecules or organelles might be segregated into an asymmetric position in the egg and embryo and maintained there are not well understood. The specification of anterior development in Drosophila can be added to the list of systems in which an asymmetrically localized cytoplasmic domain specifies the development of embryonic pattern. The molecular and genetic analysis of swallow and other genes that participate in the bicoid message localization process should bring us closer to a general understanding of the mechanism of cytoplasmic localization.

\section{Methods}

\section{Mutants}

Alleles of swallow and Df(1)JF5 are described in Gans et al. (1975), Mohler and Carroll (1984), and Stephenson and Mahowald (1987). ry $y^{506}$ is described in Coté et al. (1986). Other mutations are described in Lindsley and Grell (1966).

\section{Genomic walking}

Genomic clone B70 was isolated from the Canton S library of Maniatis et al. (1978), as described in Stephenson and Mahowald (1987). Other clones were isolated by homology to B70. $\lambda 782$ was isolated from the Canton S library, and $\lambda 528$ was isolated from a genomic library constructed by Robert Boswell from a strain of flies that he isogenized for a $c n$ bw second chromosome. Library screening, phage growth, and clone characterization were carried out according to standard procedures (Maniatis et al. 1982).

\section{Northern blots}

Total nucleic acids were extracted from staged embryos, denatured with glyoxal, electrophoresed, blotted, and hybridized, all as described previously (Stephenson and Mahowald 1987). Most Northern blots employed nick-translated restriction fragments as probes. To determine the direction of transcription of the $2.1-\mathrm{kb}$ maternally restricted RNA, the $1.3-\mathrm{kb}$ HindIII fragment from clone B70 was cloned into pGEM2 (Promega). Clones with the insert in each orientation were identified by an asymmetrically positioned restriction site. Each was digested with EcoRI, which cuts only within the multiple cloning site, and ${ }^{32} \mathrm{P}-\mathrm{la}$ beled transcripts were produced using T7 RNA polymerase (Promega), using the reaction conditions suggested by the enzyme supplier. The two probes were hybridized to Northern blot filters using the same procedures as for DNA probes.

\section{P-element transformation}

The 5.9-kb SstI-EcoRI fragment from $\lambda 528$ (see Fig. 1) was subcloned into the SstI-EcoRI sites of pUC18. The resulting plasmid, p528.1, has a Sall site at one end of the insert, because there is a SaII site in the multiple cloning site of pUC18. To create a SalI site at the other end of the insert, p528.1 was digested with EcoRI and treated with Klenow DNA polymerase to fill in the overhanging ends, and Sall linkers were added by ligation with T4 DNA ligase. The reaction was partially digested with SalI and electrophoresed, and an $8.6-\mathrm{kb}$ band was collected and eluted. This fragment was ligated at low DNA concentrations to promote recircularization and transformed into competent bacteria. The resulting plasmid, p528.11, con- tains the $5.9-\mathrm{kb}$ fragment of $\lambda 528$ with Sall sites at each end. This fragment was recloned into the Sall site of the P-element cloning vector Carnegie 20 (Rubin and Spradling 1983), and transformants containing the desired fragment were identified by colony hybridization. The $\lambda 528$-P-element construct, $\mathrm{t} 528.11$, was purified using a protocol that included banding in a $\mathrm{CsCl}-$ ethidium bromide gradient. Earlier attempts at P-element transformation using DNA that had not been purified by $\mathrm{CsCl}$ banding were unsuccessful.

Plasmids t528.11 and p $\Pi 25.7 \mathrm{wc}$ (Karess and Rubin 1984) were mixed, ethanol-precipitated, and resuspended in $5 \mathrm{~mm}$ $\mathrm{KCl}, 0.1 \mathrm{mM} \mathrm{NaPO}$ (pH 6.9), at a final concentration of 300 $\mu \mathrm{g} / \mathrm{ml}$ for $\mathrm{t} 528.11$ and $50 \mu \mathrm{g} / \mathrm{ml}$ for $\mathrm{p} \Pi 25.7 \mathrm{wc}$. Injection of preblastoderm $\mathrm{ry}^{506}$ embryos and selection of $\mathrm{ry}^{+}$transformants were as described (Rubin and Spradling 1982; Spradling and Rubin 1982; Spradling 1986).

Lines of flies with $\mathrm{ry}^{+}$inserts were established and maintained initially by manual selection for $\mathrm{ry}^{+}$at each generation. Outcrosses of $r y^{506} / r y^{506} ; \mathrm{P}\left[\left(r y^{+}, s w w^{+}\right)\right.$t528.11] males to $r y^{506 /}$ $r y^{506}$ females revealed lines with P-element inserts on the $\mathrm{X}$ chromosome. For the autosomal inserts, we crossed the P-ele-

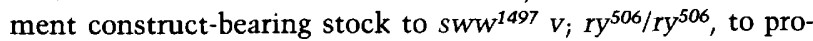
duce, in two generations, stable stocks of the genotype $s w w^{1497}$ $v / s w w^{1497} v ; r y^{506 / r y^{506}}$; $\mathrm{P}\left(\left(r y^{+}, s w w^{+}\right)\right.$t528.11]. $r y^{-}$females in these lines produce only progeny with the $s w^{-}$phenotype, whereas $r y^{+}$females produce normal progeny (see text). For the two transformed lines in which the P-element construct was inserted on the $\mathrm{X}$ chromosome, we crossed the P-element insert onto a $s w w^{11.999}$, y $c v \vee f \mathrm{X}$ chromosome in a $r y^{506 / r y^{506}}$ background, selecting for $\mathrm{ry}^{+}, \mathrm{CV}^{-}$recombinants $(\mathrm{cV}$ is only $4 \mathrm{cM}$ from $s w w$ and is thus a reasonably good visible marker for swallow), creating a stable stock of the genotype $s w w^{11-999}$, $\mathrm{P}\left[\left(r y^{+}, s w w^{+}\right) t 528.11\right] ; r y^{506 / r y^{506}}{ }^{50}$ For each of these two lines, we subsequently removed the P-element insert from the $\mathrm{X}$ chromosome by recombination, selecting for $\mathrm{ry}^{-}, \mathrm{CV}^{-}$recombinants, to confirm that the resident swallow allele was mutant.

Embryonic development was measured using females of the

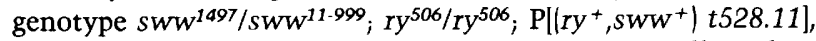
mated to an excess of Oregon R males. Eggs were collected on lightly yeasted grape juice/agar plates overnight. The eggs were counted and allowed to develop at $25^{\circ} \mathrm{C}$ for $48 \mathrm{hr}$. Rescue of the swallow ${ }^{-}$phenotype was calculated as the percentage of eggs that develop to second larval instar, avoiding the complication that some embryos of swallow ${ }^{-}$mothers hatch but die during the first larval instar.

In situ hybridization to polytene chromosomes to determine the site of insertion of the P-element construct was carried out as described (Stephenson and Mahowald 1987), using a p528.1 probe.

\section{In situ hybridizations to oocytes and embryos}

swallow probe was synthesized from plasmid g528.4, a $2.1-\mathrm{kb}$ genomic fragment in the vector pGEM3-blue (Promega). The plasmid contains a HindIII-PstI fragment between coordinates 6.0 and 8.1 in Figure 1. The plasmid was digested with HindIII and purified by phenol-chloroform extraction and ethanol precipitation. Antisense RNA was synthesized from the T7 promoter. bicoid probe was synthesized from plasmid $\mathrm{pC}^{\prime} 3-2: 2$, a 1.5-kb cDNA clone inserted in Bluescribe + (Stratagene) and provided by Mark Seeger and Thom Kaufman. The plasmid was truncated by digestion with $X b a I$ and purified as above, and the probe was synthesized from the $\mathrm{T} 7$ promoter. Transcription reactions were carried out as described (Ingham et al. 1985), using a mixture of ${ }^{35} \mathrm{~S}$-labeled thio-UTP (Amersham) and cold thioUTP to give a final concentration of $75 \mathrm{nM}$. Specific activity of 
the resulting probe was $2.2 \times 10^{8} \mathrm{dpm} / \mu \mathrm{g}$. T7 RNA polymerase was obtained from Promega or was the generous gift of Paul Kingsley. After the transcription reaction, nucleic acids were purified by phenol-chloroform extraction and ethanol-precipitation, digested with DNase I (Promega RQI DNase), phenolchloroform-extracted, and ethanol-precipitated, and the RNA probe degraded with $0.1 \mathrm{M}$ sodium carbonate $(\mathrm{pH} 10.2)$ to give a mean fragment length of about 150 nucleotides (Angerer et al. 1987). The RNA was precipitated with 2-propanol and resuspended at $0.2 \mu \mathrm{g} / \mathrm{ml}$ in the hybridization buffer described in Ingham et al. (1985).

Wild-type ovaries and embryos were from the Oregon $\mathrm{R}$ strain. swallow $w^{-}$ovaries were from females of the genotype $s w w^{11-999} / D f(1) J F 5$.

Embryos at $0-3 \mathrm{hr}$ were collected on lightly yeasted grape juice/agar plates, dechorionated in $50 \%$ commercial bleach for $5 \mathrm{~min}$, and washed in PBS [130 $\mathrm{mm} \mathrm{NaCl}, 10 \mathrm{~mm} \mathrm{NaPO}_{4} / \mathrm{pH}$ 6.5)]. Embryos were fixed for $10 \mathrm{~min}$ in a two-phase mixture of $4 \%$ formaldehyde in PBS (freshly prepared from paraformaldehyde) and heptane. The aqueous solution was removed and replaced with an equal volume of methanol. The tube was shaken vigorously, and embryos that had lost their vitelline membrane were removed from the bottom of the methanol layer and transferred to pure methanol. Embryos were rehydrated through methanol, methanol/PBS $(3: 1)$, methanol/PBS $(1: 1)$, methanol/PBS $(1: 3)$, and, finally, in pure PBS. Embryos were fixed for an additional $30 \mathrm{~min}$ in $4 \%$ formaldehyde in PBS and dehydrated, embedded, and sectioned as described below for ovaries.

Ovaries were dissected from females that had been kept for 1-2 days in an uncrowded vial with fresh yeast present. Large ovaries were teased into two or three fragments, fixed for 30 min in 4\% formaldehyde in PBS, and washed for $1 \mathrm{hr}$ in several changes of PBS. Ovaries and embryos were dehydrated and embedded using $50 \%, 70 \%, 95 \%$, and $99 \%$ ethanol, $99 \%$ ethanol/ xylene $(1: 1)$, xylene, xylene/paraffin $(1: 1)$ at $60^{\circ} \mathrm{C}$, and three changes in pure paraffin (Paraplast) at $60^{\circ} \mathrm{C}$. In our hands, ovaries become brittle if left at any stage for too long a time, and each step was typically $30-60 \mathrm{~min}$, except for the final embedding step, which was usually allowed to proceed overnight.

Five-micron sections were cut and mounted on poly-L-lysinetreated slides, and the slides were deparaffinized and treated with acetic anhydride as described (Angerer et al. 1987). We obtain adequate signals without protease treatment and have omitted this step. The RNA probes in hybridization buffer were heated at $65^{\circ} \mathrm{C}$ for $5 \mathrm{~min}$ before application to the slides, and the tissue covered with a siliconized coverslip and sealed with rubber cement. Hybridization was allowed to proceed overnight at $50^{\circ} \mathrm{C}$, and slides were washed as described in Ingham et al. (1985), a protocol that includes an RNase A treatment in $0.5 \mathrm{M}$ $\mathrm{NaCl}$. Dipping in autoradiographic emulsion and developing followed published procedures (Angerer et al. 1987). Slides were stained in $1 \%$ Giemsa stock (Banco) in $10 \mathrm{~mm} \mathrm{NaPO}_{4}(\mathrm{pH} 6.5)$ for $5 \mathrm{~min}$.

\section{Acknowledgments}

We thank Bob Boswell for his genomic library, Allan Spradling for P-element vectors and plasmids and the $r y^{506}$ stock, Mark Seeger and Thom Kaufman for the bicoid cDNA plasmid, and Paul Kingsley for T7 RNA polymerase. Kathleen Donahue provided expert technical assistance, and Michael Gagnon and Jacqueline Sterner performed preliminary versions of some of the in situ hybridizations. We are grateful to Paul Kingsley and Joanna Olmsted for instruction on injection and to Lynne and
Robert Angerer for invaluable advice on in situ hybridization. Christiane Nüsslein-Volhard provided important information prior to publication, and Drs. Lynne Angerer, Robert Angerer, and Chung-I Wu made helpful comments on the manuscript. This work was supported by grants PCM-8314455 and DCB-8702160 from the National Science Foundation.

\section{References}

Anderson, K.V. 1987. Dorsal-ventral embryonic pattern genes of Drosophila. Trends Genet. 3: 91-97.

Angerer, L.M., K.H. Cox, and R.C. Angerer. 1987. Demonstration of tissue-specific gene expression by in situ hybridization. In Guide to molecular cloning techniques (ed. S.L. Berger and A.R. Kimmell, pp. 649-661. Academic Press, New York.

Berleth, T., M. Burri, G. Thoma, D. Bopp, S. Richstein, G. Frigerio, M. Noll, and C. Nüsslein-Volhard. 1988. The role of localization of bicoid RNA in organizing the anterior pattern of the Drosophila embryo. EMBO I. 7: 1749-1756.

Coté, B., W. Bender, D. Curtis, and A. Chovnick. 1986. Molecular mapping of the rosy locus in Drosophila melanogaster. Genetics 112: 769-783.

Davidson, E.H. 1986. Gene activity in early development. Academic, New York.

Edgar, B.A. and G. Schubiger. 1986. Parameters controlling transcriptional activation during early Drosophila development. Cell 44: 871-877.

Frigerio, G., M. Burri, D. Bopp, S. Baumgartner, and M. Noll. 1986. Structure of the segmentation gene paired and the Drosophila PRD gene set as part of a gene network. Cell 47: 735-746.

Frohnhöfer, H.G. and C. Nüsslein-Volhard. 1986. Organization of anterior pattern in the Drosophila embryo by the maternal gene bicoid. Nature 324: 120-125.

- 1987. Maternal genes required for the anterior localization of bicoid activity in the embryo of Drosophila. Genes Dev. 1: 880-890.

Gans, M., C. Audit, and M. Masson. 1975. Isolation and characterization of sex-linked female-sterile mutants in Drosophila melanogaster. Genetics 81: 683-704.

Ingham, P.W., K.R. Howard, and D. Ish-Horowicz. 1985. Transcription pattern of the Drosophila gene hairy. Nature 318: 439-445.

Jeffrey, W.R. and S. Meier. 1983. A yellow crescent cytoskeletal domain in ascidian eggs and its role in early development. Dev. Biol. 96: 125-143.

Karess, R.E. and G.M. Rubin. 1984. Analysis of P transposable element functions in Drosophila. Cell 38: 135-146.

Lindsley, D.L. and E.H. Grell. 1968. Genetic variations of Drosophila melanogaster. Carnegie Inst. Washington Publ. 627.

Mahowald, A.P. and M.P. Kambysellis. 1980. Oogenesis. In The genetics and biology of Drosophila (ed. M. Ashburner and T.R.F. Wright), vol. 2c, pp. 141-224. Academic, New York.

Maniatis, T., E.F. Fritsch, and I. Sambrook. 1982. Molecular cloning: A laboratory manual. Cold Spring Harbor Laboratory, Cold Spring Harbor, New York.

Maniatis, T., R.C. Hardison, E. Lacy, J. Lauer, C. O'Connell, D. Quon, G.K. Sim, and A. Efstratiadis. 1978. The isolation of structural genes from libraries of eucaryotic DNA. Cell 15: $687-701$.

Mohler, D. and A. Carroll. 1984. Sex-linked female sterile mutants in the Iowa collection. Drosophila Inf. Serv. 60: 236241.

Nüsslein-Volhard, C., H.G. Frohnhöfer, and R. Lehmann. 1987. 
Determination of anteroposterior polarity in the Drosophila embryo. Science 238: 1675-1681.

Perrimon, N. and M. Gans. 1983. Clonal analysis of the tissuespecificity of recessive female-sterile mutations of Drosophila melanogaster using a dominant female-sterile mutation Fs(1)K1237. Dev. Biol. 100: 365-373.

Perrimon, N., D. Mohler, L. Engstrom, and A.P. Mahowald. 1986. X-linked female-sterile loci in Drosophila melanogaster. Genetics 113: 695-712.

Rubin, G.M. and A.C. Spradling. 1983. Vectors for P elementmediated gene transfer in Drosophila. Nucleic Acids Res. 11: 6341-6351.

Scharf, S.R. and J.C. Gerhart. 1983. Axis determination in eggs of Xenopus laevis: A critical period before first cleavage, identified by the common effects of cold, pressure and ultraviolet irradiation. Dev. Biol. 99: 75-87.

Spradling, A.C. 1986. P element-mediated transformation. In Drosophila, a practical approach (ed. D.B. Roberts), pp. 175-197. IRL Press, Oxford.

Spradling, A.C. and G.M. Rubin. 1982. Transposition of cloned $P$ elements into Drosophila germ line chromosomes. Science 218: $341-347$.

Stephenson, E.C. and A.P. Mahowald. 1987. Isolation of Drosophila clones encoding maternally-restricted RNAk. Dev. Biol. 124: 1-8.

Strome, S. and W.B. Wood. 1983. Generation of asymmetry and segregation of germ-line granules in early $C$. elegans embryos. Cell 35: 15-25.

Zalokar, M. 1974. Effect of colchicine and cytochalasin B on ooplasmic segregation of ascidian eggs. Wilhelm Roux's Arch. Dev. Biol. 175: 243-248.

1976. Autoradiographic study of protein and RNA formation during early development of Drosophila eggs. Dev. Biol. 49: 425-437. 


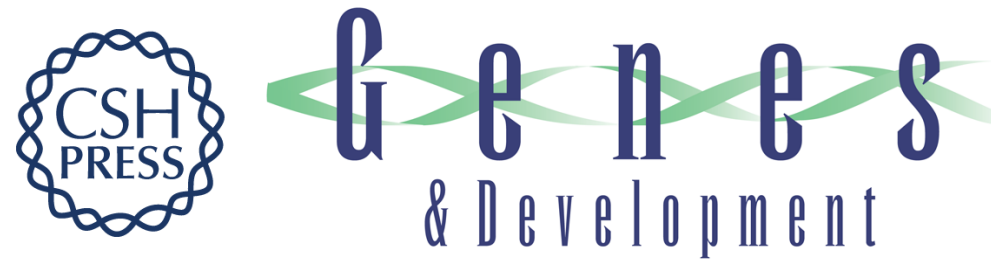

\section{Molecular analysis of the swallow gene of Drosophila melanogaster.}

E C Stephenson, Y C Chao and J D Fackenthal

Genes Dev. 1988, 2:

Access the most recent version at doi:10.1101/gad.2.12a.1655

References This article cites 23 articles, 6 of which can be accessed free at:

http://genesdev.cshlp.org/content/2/12a/1655.full.html\#ref-list-1

License

Email Alerting

Receive free email alerts when new articles cite this article - sign up in the box at the top Service right corner of the article or click here.

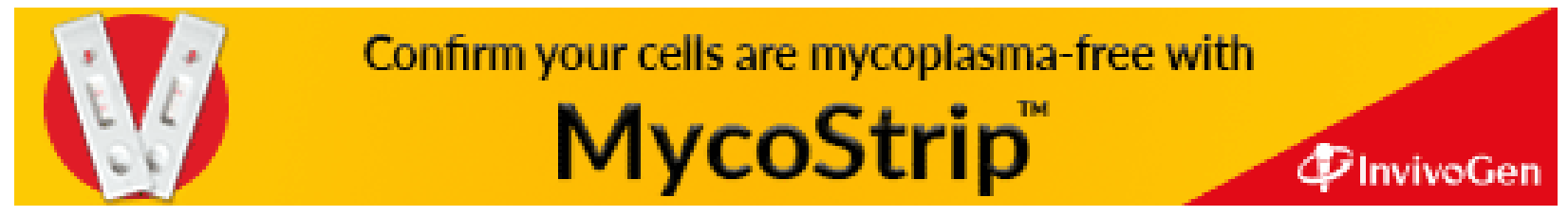

